\title{
Nucleus Accumbens Deep Brain Stimulation Produces Region-Specific Alterations in Local Field Potential Oscillations and Evoked Responses In Vivo
}

\author{
Clinton B. McCracken ${ }^{1}$ and Anthony A. Grace ${ }^{1,2,3}$ \\ Departments of ${ }^{1}$ Neuroscience, ${ }^{2}$ Psychiatry, and ${ }^{3}$ Psychology, University of Pittsburgh, Pittsburgh, Pennsylvania 15260
}

Deep brain stimulation of the nucleus accumbens (NAC) region is an effective therapeutic avenue for several psychiatric disorders that are not responsive to traditional treatment strategies. Nonetheless, the mechanisms by which DBS achieves therapeutic effects remain unclear. We showed previously that high-frequency (HF) NAC DBS suppressed pyramidal cell firing and enhanced slow local field potential (LFP) oscillations in the orbitofrontal cortex (OFC) via antidromic activation of corticostriatal recurrent inhibition. Using simultaneous multisite LFP recordings in urethane-anesthetized rats, we now show that NAC DBS delivered for 90 min at high or low frequency (LF) selectively affects spontaneous and evoked LFP oscillatory power and coherence within and between the medial prefrontal cortex (mPFC), lateral OFC, mediodorsal thalamus (MD), and NAC. Compared with LF or sham DBS, HF DBS enhanced spontaneous slow oscillations and potentiated evoked LFP responses only in OFC. HF DBS also produced widespread increases in spontaneous beta and gamma power and enhanced coherent beta activity between MD and all other regions. In contrast, LF DBS elevated theta power in MD and NAC. Analysis of acute NAC-induced oscillations showed that HF DBS increased and LF DBS decreased induced relative gamma coherence compared with sham DBS. These data suggest that HF (therapeutic) and LF (possibly deleterious) NAC DBS produce distinct region-specific and frequency band-specific changes in LFP oscillations. NAC DBS may achieve therapeutic effects by enhancing rhythmicity and synchronous inhibition within and between afferent structures, thereby normalizing function of a neural circuit that shows aberrant activity in obsessive-compulsive disorder and depression.

\section{Introduction}

Deep brain stimulation (DBS) of the nucleus accumbens (NAC) and nearby white matter tracts of the anterior internal capsule has emerged as an effective treatment strategy for a number of psychiatric disorders that fail to respond to standard pharmacological or behavioral therapies. Originally developed as a replacement for ablative surgery in the treatment of severe obsessivecompulsive disorder (OCD), long-term clinical trials have now shown that NAC DBS significantly improves symptoms in refractory OCD patients (Greenberg et al., 2009) and is also effective in treating major depression (Schlaepfer et al., 2008; Malone et al., 2009). Furthermore, case studies have suggested that NAC DBS may be indicated for treatment-resistant Tourette's syndrome (Flaherty et al., 2005; Kuhn et al., 2007b) and addiction (Kuhn et al., 2007a).

Despite this interest, relatively little is known regarding the mechanisms of action of DBS. Given the immense potential of NAC DBS for use in a variety of otherwise treatment-resistant psychiatric disorders, a better understanding of these mecha-

\footnotetext{
Received Jan. 10, 2009; revised March 11, 2009; accepted March 31, 2009.

This work was supported by National Institutes of Health Grants MH073111 (to A.A.G.) and a National Alliance for Research on Schizophrenia and Depression Young Investigator Award and Tourette Syndrome Association Fellowship (to C.B.M.). We thank N. Macmurdo for technical assistance.

Correspondence should be addressed to Clinton B. McCracken, Department of Neuroscience, A210 Langley Hall, University of Pittsburgh, Pittsburgh, PA 15260. E-mail: cbm12@pitt.edu.

D0I:10.1523/JNEUROSCI.0131-09.2009

Copyright $\odot 2009$ Society for Neuroscience $\quad 0270-6474 / 09 / 295354-10 \$ 15.00 / 0$
}

nisms is critical. Early studies of DBS for movement disorders (i.e., targeting the thalamus, globus pallidus, or subthalamic nucleus) suggested that DBS decreased activity in the stimulated area, through either depolarization blockade or enhanced local GABAergic transmission (Boraud et al., 1996; Benazzouz et al., 2000; Beurrier et al., 2001; Kiss et al., 2002); in effect, creating a functional lesion of the stimulated nucleus. However, electrical brain stimulation at clinically effective intensities preferentially excites axons as opposed to cell bodies (Nowak and Bullier, $1998 \mathrm{a}, \mathrm{b})$, indicating that activation of afferent and efferent axons, and consequent modulation of neuronal activity in sites distal to the stimulated nucleus, may contribute substantially to the therapeutic effects of DBS (Vitek, 2002; McIntyre et al., 2004; McCracken and Grace, 2007; Hammond et al., 2008).

We showed previously that NAC DBS delivered at high frequency $(130 \mathrm{~Hz}$; HF) for 30 min reduced pyramidal cell firing in the anesthetized rat orbitofrontal cortex (OFC), most likely through enhanced recurrent inhibition driven by antidromic activation of corticostriatal axon collaterals (McCracken and Grace, 2007). These changes in neuronal firing were accompanied by an enhancement of local field potential (LFP) oscillations in the slow/delta band $(0.5-4 \mathrm{~Hz})$ that developed gradually over the 30 min DBS session and an NMDA-dependent potentiation of acute NAC-evoked short-latency excitatory LFP responses in OFC. Low-frequency $(10 \mathrm{~Hz}$; LF) stimulation failed to produce any changes in LFP activity. The frequency dependence of these changes in LFP activity is noteworthy; in the clinic LF DBS for 
A.

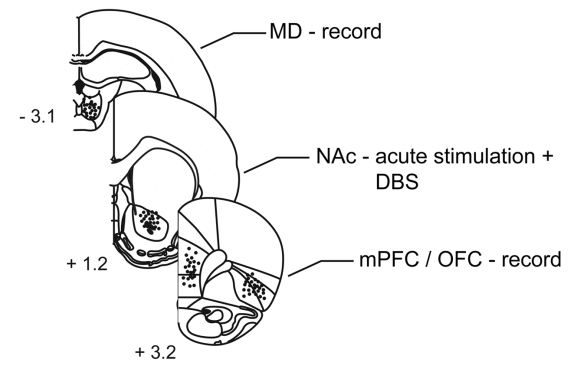

B.

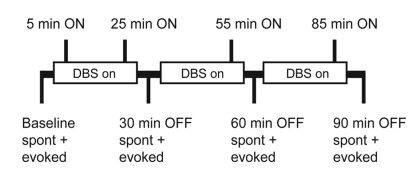

Figure 1. Stimulation and recording protocol. $\boldsymbol{A}$, Representative nonoverlapping electrode placements in the mediodorsal thalamus, nucleus accumbens, and medial prefrontal and orbitofrontal cortices. $\boldsymbol{B}$, Schematic of experimental design. LFP activity was sampled with DBS off after 30,60 , and 90 min of stimulation and also with DBS on at discrete time points.

A.

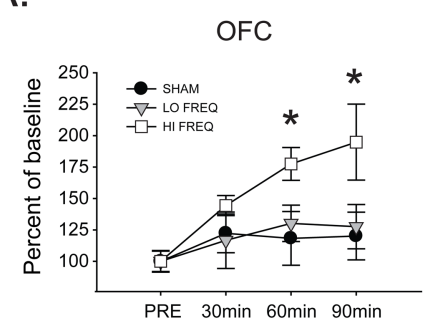

Slow/delta $(0.5-4 \mathrm{~Hz})$
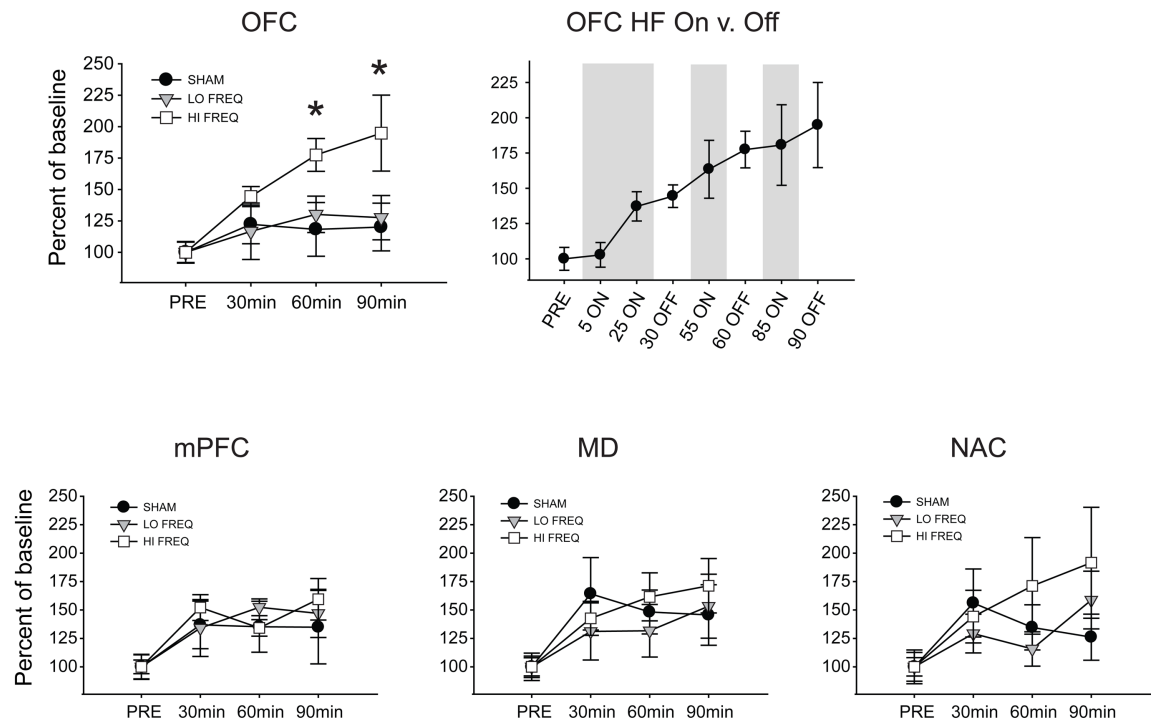

B.
OFC delta: pre-HF DBS

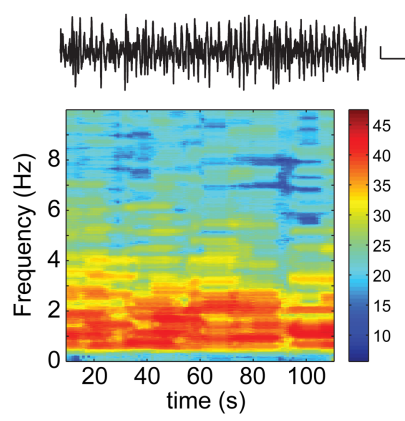

OFC delta: post-90 min HF DBS
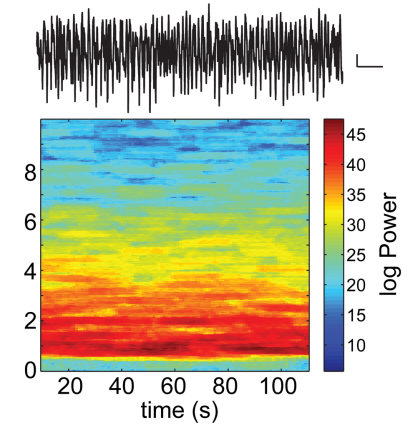

Figure 2. HF stimulation produces a time-dependent increase in slow/delta oscillations selectively in the OFC. $A$, HF NAC DBS produces a time-dependent enhancement of slow oscillations ( $0.5-4 \mathrm{~Hz})$ in OFC compared with LF and SHAM DBS (top left). Analysis of LFP activity with HF DBS on demonstrates that this effect was not apparent early in the stimulation period, but was maintained during the "off" period (top right). There were no changes in delta oscillations in the other regions with any stimulation frequency (bottom). * Significantly different from LF and SHAM. B, Representative OFC voltage traces and time-frequency spectrograms before and after 90 min HF NAC DBS showing increased low frequency activity following $90 \mathrm{~min}$ DBS. Calibration: $5 \mathrm{~s}, 0.2 \mathrm{mV}$.

most indications generally does not produce therapeutic effects and may in fact be deleterious (Moro et al., 2002; Kupsch et al., 2003; Timmermann et al., 2004; Kuncel et al., 2007; Cooper et al., 2008; Florin et al., 2008), although more recent evidence suggests that in some conditions (i.e., pedunculopontine stimulation for Parkinson's disease), LF stimulation may be beneficial (Nandi et al., 2008). Here, we extend our previous findings by examining how NAC DBS delivered for $90 \mathrm{~min}$ at high and low frequencies affects spontaneous and evoked LFP oscillatory power and coherence within and between the medial prefrontal cortex (mPFC), lateral OFC, mediodorsal thalamus (MD), and NAC; regions comprising a circuit that often exhibits aberrant metabolism in patients suffering from OCD or major depression (Deckersbach et al., 2006; Evans et al., 2006).

\section{Materials and Methods}

All procedures were performed in accordance with the guidelines outlined in the NIH Guide for the Care and Use of Laboratory Animals, and were approved by the Institutional Animal Care and Use Committee of the University of Pittsburgh.

\section{Animals and surgery}

Male Sprague Dawley rats (275-400 g) were anesthetized with urethane $(1.5 \mathrm{~g} / \mathrm{kg}$, i.p.) and placed in a stereotaxic frame. Body temperature was maintained at $37^{\circ} \mathrm{C}$ with a temperaturecontrolled heating pad. In all surgical preparations the scalp was exposed and burr holes were drilled in the skull overlying the lateral OFC, mPFC, the NAC core, and the MD. Concentric bipolar stimulating electrodes (NEX-100; Kopf) were placed in the NAC-anteroposterior (AP) $+1.2 \mathrm{~mm}$ (from bregma), mediolateral (ML) $+2.0 \mathrm{~mm}$, dorsoventral (DV) -6.9 $\mathrm{mm}$ (from skull); Teflon-insulated stainlesssteel recording electrodes $(0.1 \mathrm{~mm}$ diameter, Plastics One) were slowly lowered into the OFC (AP: $+3.2 \mathrm{~mm}$, ML: $+3.4 \mathrm{~mm}$, DV: $-5.5 \mathrm{~mm}$ ), mPFC (AP: $+3.2 \mathrm{~mm}, \mathrm{ML}:+0.7 \mathrm{~mm}, \mathrm{DV}:-4$ $\mathrm{mm}$ ), and $\mathrm{MD}$ (AP: $-3.3 \mathrm{~mm}$, ML: $+0.7 \mathrm{~mm}$, DV: $-5.5 \mathrm{~mm}$ ). Recording commenced no earlier than 30 min following electrode implantation.

\section{Recording}

LFP signals from the recording electrodes were amplified (gain: 1000), analog filtered (0.1-500 $\mathrm{Hz}$ ) by a multichannel amplifier (A-M Systems) and displayed on an oscilloscope (Tektronics). The data were digitized at $1 \mathrm{kHz}$ and acquired using custom-designed computer software (Neuroscope) and stored for off-line analysis. The animal ground was used as a reference for LFP recordings. DBS was applied to separate groups at $130 \mathrm{~Hz}$ (HF) or $10 \mathrm{~Hz}$ (LF) using standard parameters $(0.2 \mathrm{~mA}, 0.1 \mathrm{~ms}$ pulse duration). Another group had the DBS electrode implanted but was not stimulated (SHAM). DBS was applied for three $30 \mathrm{~min}$ sessions for a total of $90 \mathrm{~min}$; sessions were separated by 5 min intervals where spontaneous and evoked LFP data were sampled with DBS OFF. For spontaneous recordings, LFPs were recorded for $2 \mathrm{~min}$ at a number of different time points with DBS ON or OFF-pre-DBS baseline (OFF), during the first $5 \mathrm{~min}$ of DBS (ON), after $25 \mathrm{~min}$ of DBS (ON), after 30 min of DBS (OFF), after $55 \mathrm{~min}(\mathrm{ON}), 60 \mathrm{~min}(\mathrm{OFF}), 85 \mathrm{~min}(\mathrm{ON})$, and 
$90 \mathrm{~min}$ (OFF). Figure 1 shows a schematic of the recording procedure. During OFF periods, spontaneous LFP data were recorded, followed immediately by evoked data, after which DBS was turned ON again. For evoked LFP responses, baseline input-output curves were generated using 4 stimulus intensities $(0.2,0.4,0.8,1.2 \mathrm{~mA}, 0.2 \mathrm{~ms}$ pulse duration, 20 stimulation sweeps at $0.4 \mathrm{~Hz}$ for each intensity). LFP data were recorded for $0.2 \mathrm{~s}$ before stimulus and $1 \mathrm{~s}$ after stimulus. Evoked activity was reassessed following 30,60 , and 90 min of DBS.

Multisite LFP data were recorded from a total of 33 animals (11/ group). Stimulating electrodes were located in the NAC core (one pole of the stimulating electrode was used to record spontaneous NAC LFP activity when stimulation was off), OFC recording electrodes in the lateral OFC and ventral agranular insular cortex, mPFC electrodes in the prelimbic and infralimbic cortices, and MD electrodes in the mediodorsal thalamus (Fig. 1). All data from animals with incorrectly placed NAC stimulating electrodes were excluded; for the other regions data were excluded on a region by region basis, leaving final group sizes of 7-10 animals/group. Spontaneous and evoked LFP activity were sampled before DBS, and after 30,60, and 90 min of SHAM (implanted but not stimulated), LF or HF stimulation. "Spontaneous" LFP activity was also recorded during DBS ON; after 1, 25, 55, and 85 min of LF or HF stimulation (see Fig. 1 for schematic). However, in the LF group, there were acute evoked responses from the stimulation that irrevocably contaminated the analysis of oscillatory activity with DBS ON. Accordingly, all statistical comparisons between SHAM, LF, and HF groups were made during the stimulation off periods.

\section{Analysis}

The spectral power of LFP oscillations in each region and coherence between regions was analyzed using routines from the Chronux software package (www.chronux.org) for Matlab (MathWorks). Chronux uses a multitaper method of spectral analysis, where data are multiplied by a specified number of orthogonal tapers, before applying a fast Fourier transform (FFT) to the tapered waveforms. The transformed spectral data are then averaged over tapers as a way of reducing variance and bias. These methods have now been successfully applied to neural data in a number of cases (Mitra and Pesaran, 1999; Pesaran et al., 2002; Womelsdorf et al., 2006; DeCoteau et al., 2007). To assess coherence between simultaneously recorded signals, the FFTs of the tapered waveforms were calculated individually for each taper. Cross-spectra of the signals were derived from the FFTs for each taper and trial and then were averaged over tapers. Coherence was then calculated as $C=(\mathrm{S} 12) / \sqrt{\mathrm{S} 1 \times \mathrm{S} 2}$, where S12 represents the average cross-spectral density and S1 and S2 represent the average power spectral density of the two signals. For spontaneous LFP data, recordings were downsampled at $200 \mathrm{~Hz}$ and segmented ( $10 \mathrm{~s}$ window). Each segment was detrended to remove any slow DC components and padded with zeros to increase frequency resolution.

Multitaper spectral power and coherence were calculated for each segment in the following frequency bands: slow/delta $(0.5-4 \mathrm{~Hz})$; theta $(4-12 \mathrm{~Hz})$; beta $(13-30 \mathrm{~Hz})$; and gamma $(30-70 \mathrm{~Hz}$, not including $58-62 \mathrm{~Hz}$ to avoid line noise). Data for each frequency band were then averaged over segments. To compare across different groups, power and coherence values for each animal in a group were normalized to the mean baseline values for that group and are referred to as "relative power" and "relative coherence".

Evoked responses. Acute NAC stimulation produced a negative-going short-latency peak in OFC and mPFC termed N1; N1 amplitude was defined as the peak voltage deflection subtracted from the voltage value $0.5 \mathrm{~ms}$ before stimulation.

Induced oscillations. Induced oscillatory activity was calculated using the two higher stimulation intensities. Spectral power and coherence values in the gamma band in the $1 \mathrm{~s}$ after stimulus were normalized to prestimulus baseline $(200 \mathrm{~ms})$ and averaged across stimulation sweeps for each time point. To compare across different groups, the values for each animal in a group were normalized to the mean baseline values for that group.

\section{Statistics}

Changes in spontaneous and evoked power in each region and coherence between regions due to time and DBS frequency were analyzed using
A. Theta $(4-12 \mathrm{~Hz})$
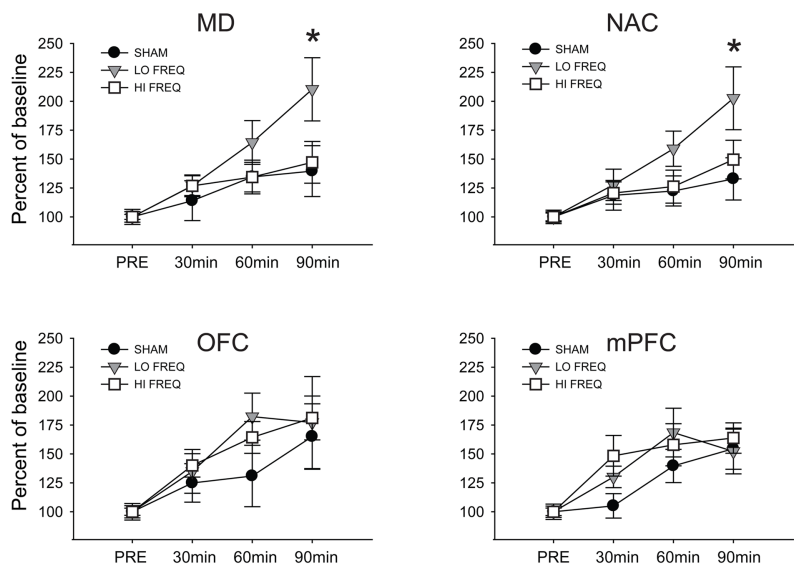

B.
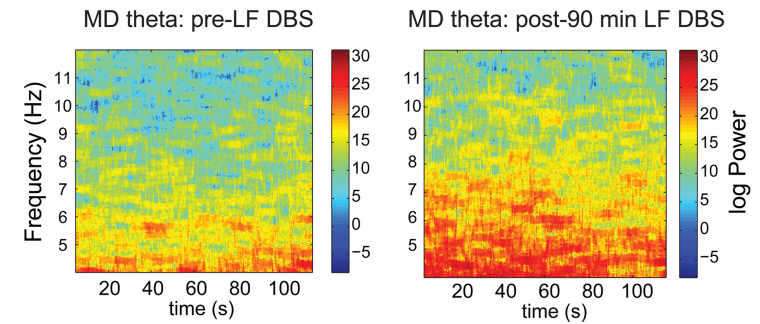

Figure 3. Only LF stimulation affected theta activity. $\boldsymbol{A}$, LF NAC DBS produces a timedependent increase in theta oscillation power $(4-12 \mathrm{~Hz})$ in $\mathrm{MD}$ and $\mathrm{NAC}$ compared with $\mathrm{HF}$ and SHAM DBS. *Significantly different from HF and SHAM. $\boldsymbol{B}$, Representative MD time-frequency spectrograms before and after 90 min LF NAC DBS show increases in theta band activity.

two-way ANOVA with time as a repeated measure and Holm-Sidak post hoc test. Changes in N1 amplitude were calculated using two-way ANOVA with time and stimulation intensity as repeated measures, and Holm-Sidak post hoc test. Significance was set at $p<0.05$.

\section{Results}

\section{Spontaneous oscillatory activity}

Power

In OFC, there was a time-dependent increase in slow/delta power with HF stimulation that was apparent after 60 and 90 min of stimulation [significant main effects of time $\left(F_{(3,69)}=7.98, p<\right.$ $0.001)$ and frequency $\left(F_{(2,69)}=3.813, p=0.039\right)$, but no significant interaction $\left.\left(F_{(6,69)}=1.693, p=0.138\right)\right]$ (Fig. $\left.2 A\right)$.

Comparison of DBS ON versus OFF in the HF group (Fig. $2 A$ ) shows that these effects developed over time with no acute effects of stimulation, such that 5 min ON was not significantly different from baseline, and $85 \mathrm{~min}(\mathrm{ON})$ was not significantly different that $90 \mathrm{~min}$ (OFF). In all other brain regions (mPFC, MD, NAC), there was no significant effect of HF DBS on slow/delta power compared with SHAM or LF stimulation (significant main effect of time; $F>6.61, p<0.001$ in all cases, but post hoc analysis revealed no significant differences between SHAM, LF, and HF groups at any time point in any region) (Fig. $2 \mathrm{~A}$ ).

Theta $(4-12 \mathrm{~Hz}$ ). In both MD and NAC (Fig. 3A), theta power in the LF groups was significantly increased compared with both SHAM and HF groups after 90 min of stimulation [main effects of time ( $F>8.495, p<0.001$ in both regions), but not frequency $(F<1.597, p>0.223$ in both region), and no interaction effects $(F<1.458, p>0.255$ in both regions $)]$. In contrast, in OFC and mPFC (Fig. $3 A$ ), neither HF nor LF stimulation produced any effects that were significantly different from SHAM [significant 
A.
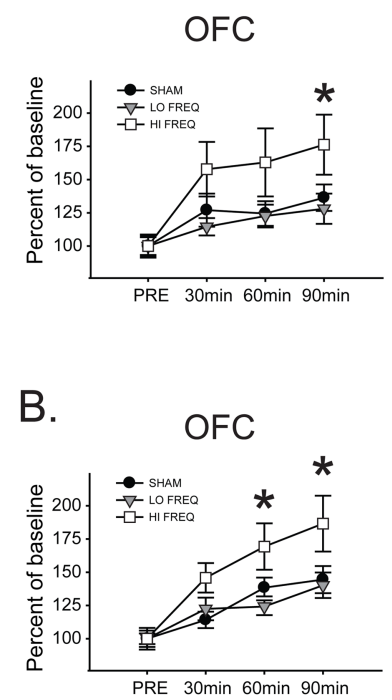

Beta $(13-30 \mathrm{~Hz})$

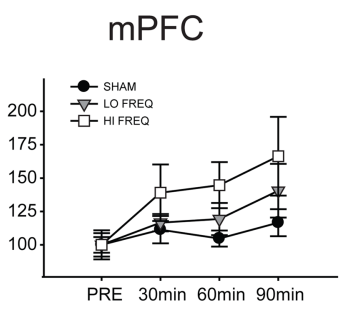

MD

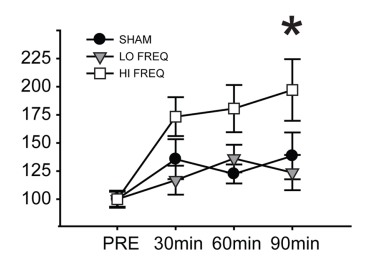

NAC

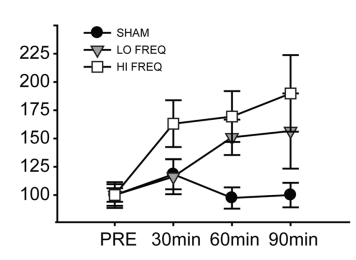

Gamma $(30-70 \mathrm{~Hz})$
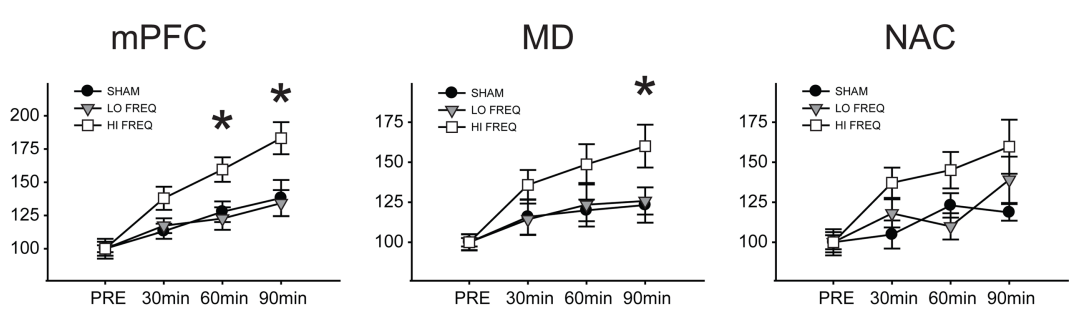

\section{C.}
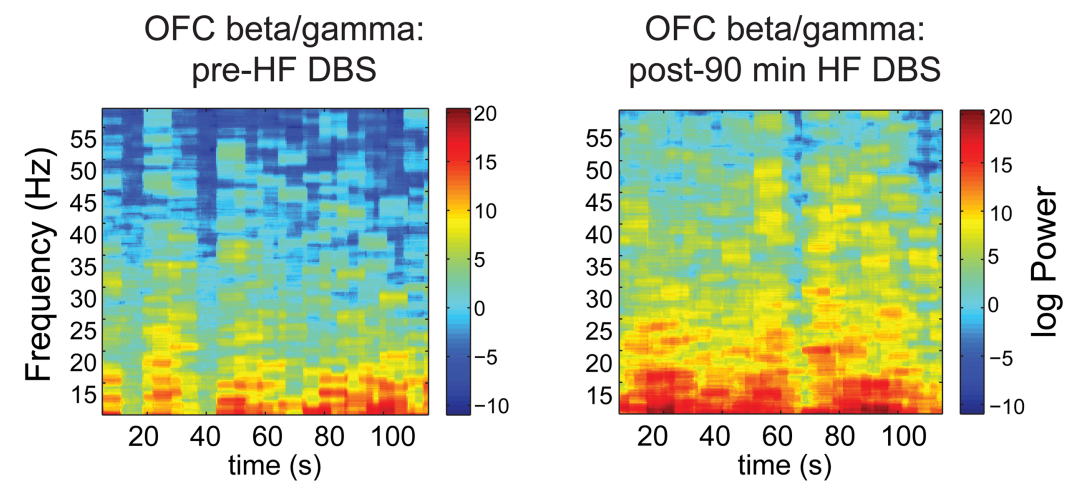

Figure 4. HF stimulation causes widespread increases in beta and gamma band activity. $A, H F N A C D B S$ significantly enhances beta activity (13-30 Hz) in OFC and MD compared with LF and SHAM DBS. B, HF NAC DBS increases gamma oscillation ( $30-70 \mathrm{~Hz}$ ) power in OFC, mPFC, and MD compared with LF and SHAM DBS. *Significantly different from HF and SHAM. C, Representative OFC time-frequency spectrograms before and after 90 min HF NAC DBS showing increases in beta and gamma band activity in this region.

main effects of time $(F>19.44, p<0.001$ in both regions $)$, with no significant effects of frequency $(F<0.739, p>0.488$ in both cases) and no significant interactions $(F<1.235, p>0.298$ in both cases); post hoc tests showed no difference between any groups at any time point in either region].

Beta $(13-30 \mathrm{~Hz})$. In OFC and MD, there were significant increases in beta power in the HF group (Fig. 4A) compared with SHAM and LF groups after 90 min of stimulation [main effects of time $(F>9.781, p<0.001$ in both cases $)$ but not frequency $(F<$ 1.597, $p>0.223$ in both cases) and no significant interactions $(F<1.969, p>0.084$ in both cases)]. In the NAC (Fig. $4 A)$, both LF and HF stimulation increased beta power compared with sham after 60 and $90 \mathrm{~min}$ of stimulation [main effect of time $\left(F_{(3,75)}=6.232, p<0.001\right)$, but not frequency $\left(F_{(2,75)}=3.158\right.$,

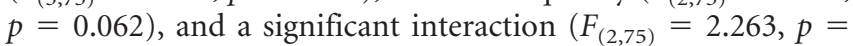
$0.048)]$. There were no significant differences in beta power in $\mathrm{mPFC}$ between groups at any time point.

Gamma $(30-70 \mathrm{~Hz})$. In OFC and mPFC, there were significant increases in spontaneous gamma power in the HF group (Fig. $4 B$ ) compared with SHAM and LF groups after 60 and 90 min of stimulation [main effects of time $(F>24.006, p<0.001$ in both cases) and frequency ( $F>4.475, p<0.024$ in both cases) and significant interaction effects $(F>2.861, p<0.016$ in both cases)] and in MD (Fig. 4A) after 90 min of stimulation [main effect of time $\left(F_{(3,66)}=14.325, p<0.001\right)$ but not frequency $\left(F_{(2,66)}=2.311, p=0.125\right)$ and no significant interaction $\left(F_{(6,66)}\right.$ $=1.445, p=0.213$ )]. In NAC (Fig. $4 A$ ), gamma power in the HF group was significantly increased compared with LF (but not SHAM) after $60 \mathrm{~min}$ of stimulation and significantly increased compared with SHAM (but not LF) after 90 min of stimulation [main effect of time $\left(F_{(3,75)}=13.051, p<0.001\right)$ but not frequency $\left(F_{(2,75)}=2.985, p=0.071\right)$, and no interaction $\left(F_{(6,75)}=\right.$ 2.009, $p=0.077)]$.

Thus, HF DBS enhanced slow oscillations over time only in $\mathrm{OFC}$, and also produced widespread time-dependent increases in beta (OFC and MD) and gamma (OFC, mPFC, and MD) power. In contrast, LF DBS produced specific, time-dependent elevations in theta power in MD and NAC.

\section{Relative coherence}

There were no main effects of time $(F<1.959, p>0.133$ in all cases) or frequency $(F<1.959, p>0.133$ in all cases $)$ and no interaction effects $(F<1.726, p>0.135$ in all cases $)$ on spontaneous relative coherence in the slow and theta bands between any regions (data not shown).

Beta. There were no significant differences in relative OFC- 
mPFC beta coherence between SHAM, LF, and $\mathrm{HF}$ groups at any time point (Fig. $4 A$ ), whereas $\mathrm{OFC}-\mathrm{MD}$ relative beta coherence (Fig. 5A) was significantly increased in the HF group compared with SHAM and LF after $60 \mathrm{~min}$ of stimulation and significantly increased compared with LF (but not SHAM) after 90 min of stimulation [main effect of time $\left(F_{(3,60)}=3.421, p=\right.$ $0.023)$, but not frequency $\left(F_{(2,60)}=3.009\right.$, $p=0.072)$, and a significant interaction $\left.\left(F_{(6,60)}=2.487, p=0.032\right)\right]$. In addition, relative beta coherence between both $\mathrm{PF}$ C-MD and MD-NAC (Fig. 5A) was significantly increased in the HF group compared with SHAM and LF groups after 60 and 90 min of stimulation [main effects of time $(F>2.816, p<0.048$ in both cases $)$ and frequency $(F>5.391, p<0.015$ in both cases) and significant interaction effects $(F>2.467, p<0.036$ in both cases $)$. In contrast, there were no changes in relative beta coherence between OFC-NAC (Fig. 4C) or PFC-NAC (Fig. 4E).

Gamma. MD-NAC gamma coherence was significantly elevated in the HF group compared with SHAM and LF groups after 60 min of stimulation [main effect of time $\left(F_{(3,57)}=4.943, p=0.004\right)$, but not frequency $\left(F_{(2,57)}=5.631, p=0.062\right)$, and no interaction $\left.\left(F_{(6,67)}=2.014, p=0.081\right)\right]$. There were no significant changes in gamma coherence between any other regions at any time point (data not shown).

Thus, the major effects of HF DBS were specific increases in coherent beta activity between $\mathrm{MD}$ and all other regions (OFC, $\mathrm{mPFC}$, and NAC), suggesting increased synchrony of fast rhythmic thalamocortical activity associated with interneuron networks.

\section{Evoked LFP activity-amplitude}

Acute NAC stimulation produced robust short-latency LFP responses in OFC and mPFC (Fig. 6), with average N1 latencies of $5.33 \pm 0.08 \mathrm{~ms}$ and $3.91 \pm 0.17 \mathrm{~ms}$, respectively. We showed previously that NAC-evoked LFP responses in OFC had distinct glutamatergic and GABAergic components that were likely due to antidromic activation of recurrent collaterals, and that N1 amplitude was potentiated following $30 \mathrm{~min}$ of HF but not LF NAC DBS (McCracken and Grace, 2007). NAC-evoked responses in $\mathrm{MPFC}$ were qualitatively similar, and since both OFC and mPFC project to the NAC core stimulation site (Gabbott et al., 2005), these responses are likely analogous to those recorded in OFC. We did not observe reliable short-latency responses to acute NAC stimulation in MD (i.e., no N1) and as such did not assess the effects of DBS on NAC evoked responses in this region.

The effects of NAC DBS on evoked LFP responses were assessed by constructing input-output curves with four stimulation intensities before DBS, and after 30,60, and 90 min of stimulation (Fig. 6A). N1 amplitude recorded in the OFC using the two higher stimulation intensities was significantly increased compared with baseline following 60 and $90 \mathrm{~min}$ of stimulation [significant main effects of time $\left(F_{(3,69)}=4.043, p=0.020\right)$ and intensity $\left(F_{(3,69)}=86.926, p<0.001\right)$, and a significant interaction effect $\left.\left(F_{(9,69)}=4.275, p<0.001\right)\right]$. In contrast, for both SHAM and LF groups there were no significant differences be- tween time points at any stimulation intensity for either group. In mPFC (Fig. 6B), there were no significant differences between time points at any stimulation intensity for any group.

\section{Stimulus-induced gamma activity-power and coherence}

Acute NAC stimulation frequently resulted in induced oscillations (i.e., not time locked to the stimulus; see Fig. 7A) in all recording sites, particularly at the higher acute stimulation intensities ( 0.8 and $1.2 \mathrm{~mA})$. Changes in induced oscillations as a function of time and stimulation frequency were quantified by examining the spectral power within regions and coherence between regions in the $1 \mathrm{~s}$ following stimulation, normalized to activity in the $200 \mathrm{~ms}$ before stimulation. Analysis was restricted to the gamma band due to the relatively short prestimulus baseline.

\section{Induced gamma power}

Induced gamma activity in the SHAM and HF groups was significantly reduced compared with the LF group (but was not different between SHAM and HF) in both OFC and mPFC (Fig. 7B) following 30, 60, and 90 min of stimulation [main effect of time in $\operatorname{mPFC}\left(F_{(3,66)}=33.727, p<0.001\right)$ but not OFC $\left(F_{(3,69)}=1.609\right.$, $p=0.193)$, main effects of frequency in both regions $(F>4.649$, $p<0.017$ in both cases), and significant interactions in both regions $(F>8.44, p<0.001$ in both cases $)]$. No significant differences were found in MD between SHAM, LF, and HF groups at any time point (Fig. $7 B$ ).

\section{Induced gamma coherence}

Induced relative gamma coherence between both OFC-PFC and OFC-MD (Fig. 7C) was significantly elevated in the HF group compared with LF (but not SHAM) after $30 \mathrm{~min}$ of stimulation. Following $60 \mathrm{~min}$ of stimulation, relative OFC-MD coherence was significantly increased in the HF group compared with LF 
A.

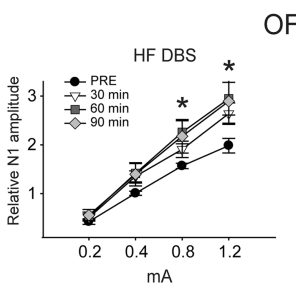

OFC
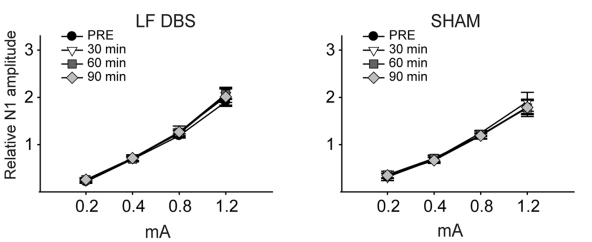

B.

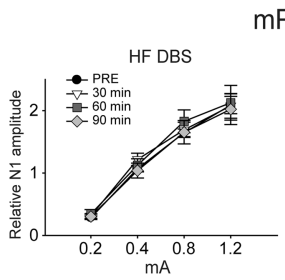

mPFC
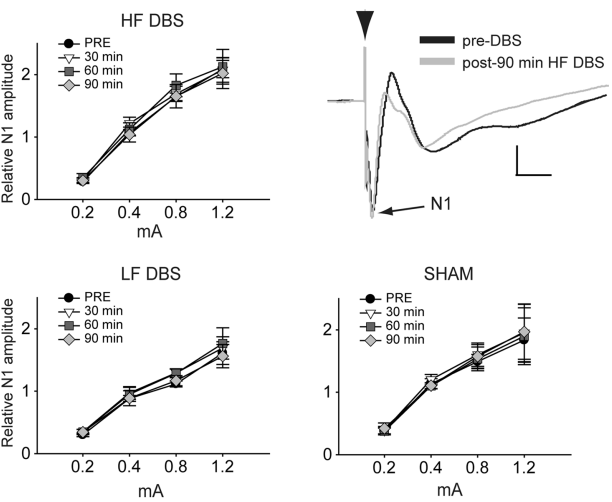

Figure 6. HF DBS selectively potentiates short-latency evoked responses in OFC. $\boldsymbol{A}, \mathrm{HF} N \mathrm{NAC}$ DBS produces a time-dependent potentiation of NAC-evoked short-latency LFP responses (N1 amplitude) in OFC. LF or SHAM DBS failed to alter evoked LFP responses in OFC. Traces show average of five stimulation sweeps of NAC-evoked OFC responses before and after $90 \mathrm{~min} \mathrm{HF}$ NAC DBS. *Significantly different from LF and SHAM. Calibration: $20 \mathrm{~ms}, 0.2 \mathrm{mV}$. $\boldsymbol{B}$, NAC DBS did not produce any effects at any frequency on NAC-evoked LFP responses in mPFC. Traces show average of five stimulation sweeps of NAC-evoked mPFC responses before and after $90 \mathrm{~min} \mathrm{HF}$ NAC DBS. Calibration: $20 \mathrm{~ms}, 0.2 \mathrm{mV}$.

and SHAM, and after 90 min of stimulation, relative coherence between OFC-PFC and OFC-MD was significantly higher in the HF group than in either the LF or the SHAM group [main effects of time ( $F>2.777, p<0.047$ in both cases) and frequency $(F>$ $3.407, p<0.044$ in both cases) and significant interactions $(F>$ $4.208, p<0.001$ in both cases)]. Furthermore, relative gamma coherence was significantly decreased between OFC-MD and OFC-mPFC in the LF group compared with HF and SHAM groups following $90 \mathrm{~min}$ of LF stimulation (Fig. 7C). Induced relative PFC-MD gamma coherence (Fig. $7 C$ ) was significantly elevated in the HF groups compared with the LF and SHAM groups after 30,60, and 90 min of stimulation [main effect of time $\left(F_{(3,54)}=16.636, p<0.001\right)$ and frequency $\left(F_{(2,54)}=7.084, p=\right.$ $0.003)$ and a significant time $\times$ frequency interaction $\left(F_{(6,54)}=\right.$ $6.882, p<0.001)]$.

Thus, HF DBS produced a significant increase and LF DBS a significant decrease in coherent gamma activity between OFCPFC and OFC-MD. This effect suggests that HF DBS leads to coordinated induced activity in the gamma band. The opposite effect of LF DBS is particularly noteworthy given the divergent effects of HF and LF DBS in the clinic.

\section{Discussion}

NAC DBS delivered for 90 min produced widespread changes in spontaneous and evoked LFP oscillations in brain regions with known involvement in obsessive-compulsive disorder, and affected coordinated activity (coherence) between these regions. Many of these changes occurred in a region- and frequency bandspecific manner, and were strongly dependent on stimulation frequency such that HF and LF DBS each produced a unique constellation of effects on rhythmic LFP activity (Fig. 8).

The major findings specific to HF (i.e., therapeutic) DBS include a time-dependent enhancement of spontaneous slow oscillatory activity specific to OFC, and time-dependent increases in fast oscillatory activity in a number of regions. These withinregion changes in rhythmic activity were accompanied by HF DBS-specific increases in spontaneous and stimulus-induced coherent activity between regions in the higher frequency bands, particularly in the thalamocortical system. In the clinic, successful outcomes have been achieved targeting both white matter tracts of the anterior internal capsule (Greenberg et al., 2009; Malone et al., 2009) and NAC gray matter itself (Sturm et al., 2003; Schlaepfer et al., 2008). While stimulation of one target (e.g., NAC gray matter) likely affects the other (capsular white matter) to some extent due to current spread, our data suggest that the one common element across all studies is activation of descending cortical fibers that either traverse (in the context of white matter) or terminate in NAC. Indeed, chronaxie studies indicate that the brief stimulus pulse duration would favor activation of myelinated fibers rather than neuronal somata (Ranck, 1975; Nowak and Bullier, 1998a).

\section{Significance of changes in LFP activity}

Rhythmic oscillatory fluctuations in LFP recordings arise from synchronous activity in relatively large groups of neurons (Mitzdorf, 1985; Logothetis, 2003) and have been characterized in a number of brain regions. LFP oscillations in discrete frequency bands correspond to behavioral states across the sleepwake cycle, in addition to particular aspects of neural computation (Steriade, 2006). Mechanistic interpretations of changes in LFP oscillations as they relate to cognitive processes in anesthetized rodents are challenging. Nonetheless, both spontaneous and evoked/induced oscillations have been characterized in brain slices and anesthetized animals. Slow/delta oscillations are prominent in sleep and anesthesia (Steriade et al., 1993; Cowan and Wilson, 1994), and theta, beta, and gamma oscillations have all been characterized in anesthetized or in vitro preparations (Kamondi et al., 1998; Faulkner et al., 1999; Neville and Haberly, 2003; Hajós et al., 2008; Yamawaki et al., 2008). Drawing from these data, analyses of the effects of DBS on rhythmic LFP activity can shed light on how DBS affects basic mechanisms of neural synchronization within and between regions. Furthermore, LFP activity correlates with blood-flow-dependent imaging methods to a greater degree than do measures of neuronal spiking (Logothetis et al., 2001; Maier et al., 2008) and therefore provide a better translational index of activity states when comparing effects of DBS in animals and humans. Indeed, acute HF NAC DBS in OCD patients produced cerebral blood flow changes that correspond well to the pattern of changes in LFP activity observed here (Rauch et al., 2006).

\section{DBS effects on spontaneous LFP oscillations}

Slow/delta LFP oscillations are proposed to represent synchronous changes in membrane potential produced by recurrent activity (Steriade et al., 1993; Sanchez-Vives and McCormick, 2000; Shu et al., 2003). We showed previously that HF but not LF NAC DBS increased slow/delta oscillations in OFC; an effect that was not apparent immediately after stimulation began but developed 
over 30 min of DBS (McCracken and Grace, 2007). We show here that this effect is specific to OFC and demonstrates a progressive increase in magnitude over the 90 min stimulation period. There are two likely possibilities for the observed regional specificity of this effect: (1) the OFC sends comparatively denser projections to the NAC stimulation site, and therefore is more strongly activated. Thus, the enhanced slow oscillations in the OFC may be due to increased recurrent activity stemming from preferential antidromic activation of OFC projection neuron recurrent collaterals (McCracken and Grace, 2007); alternately, (2) the specific changes in slow oscillations are due to regional differences in intrinsic network properties. Indeed, recent studies suggest that $\mathrm{mPFC}$ and OFC respond differently to similar stimuli (Crombag et al., 2000; Homayoun and Moghaddam, 2006; Moghaddam and Homayoun, 2008).

Theta oscillations in the rodent, best studied in the hippocampus (Buzsáki, 2002), have also been identified in prefrontal cortex (Paz et al., 2008) and striatum (DeCoteau et al., 2007), and are involved in spatial navigation and mnemonic processes. LF NAC DBS enhanced theta activity in MD and NAC, similar to that reported for in vitro cortical preparations, where $\mathrm{LF}(4 \mathrm{~Hz})$ stimulation promoted theta activity (Yamawaki et al., 2008). The significance of these changes is unclear; however, LF DBS for movement disorders often produces deleterious effects in the clinic (Moro et al., 2002; Kupsch et al., 2003; Cooper et al., 2008; Florin et al., 2008) and thus the enhanced theta power in these regions may represent a physiological correlate of a pathological state. Supporting this idea, patients with OCD display higher theta activity than controls (Karadag et al., 2003; Desarkar et al., 2007), and furthermore, NAC stimulation at $\sim 10 \mathrm{~Hz}$ increased compulsive-like behavior in a rodent model of OCD (van Kuyck et al., 2003).

Fast oscillations in the beta and gamma bands occur in response to sensory stimuli (Barth and MacDonald, 1996; TallonBaudry, 2003) and are associated with higher-order cognitive processes including attention and perception (Singer, 1993; Farmer, 1998; Schnitzler and Gross, 2005). On a cellular level, fast rhythmic activity is generated via synchronous inhibition within networks of GABAergic interneurons, with oscillation frequency and duration modulated by glutamatergic input (Traub et al., 2004). The exact relationship between beta and gamma generation remains unclear. In vivo and in vitro studies suggest that stimulus-elicited gamma oscillations are often followed immediately by beta oscillations (Traub et al., 1999; Haenschel et al., 2000); however, these rhythms may also coexist simultaneously (Bressler, 1984; Roopun et al., 2006). Computational studies indicate that while both rhythms promote synchronization, beta activity is synchronizing over relatively longer distances and time scales compared with gamma (Kopell et al., 2000). HF DBS pro- duced widespread increases in spontaneous beta and gamma power and enhanced fast coherent thalamocortical activity. This enhanced fast oscillatory synchronization may stem from LTPmediated increased drive onto interneurons in afferent regions produced by NAC DBS (McCracken and Grace, 2007). Within the context of OCD, the enhancement of beta activity by DBS may have important functional implications. EEG studies suggest that OCD patients have lower beta activity than healthy controls (Kuskowski et al., 1993; Serra et al., 1994; Karadag et al., 2003; Pogarell et al., 2006), fail to exhibit the enhanced beta activity to olfactory stimulation seen in controls (Locatelli et al., 1996), and show lower levels of postmovement beta synchronization (Leocani et al., 2001). Sensory-evoked beta oscillations have been linked to novelty detection (Kisley and Cornwell, 2006). Thus, although speculative, increasing beta activity in OCD patients may enhance salience of external stimuli, thereby interrupting ritualistic patterns and promoting reengagement of other behaviors.

\section{DBS effects on induced LFP oscillations}

While spontaneous measures represent the general activity state of the system, stimulated responses provide an index of responsivity of the system to activation, such as in the process- 


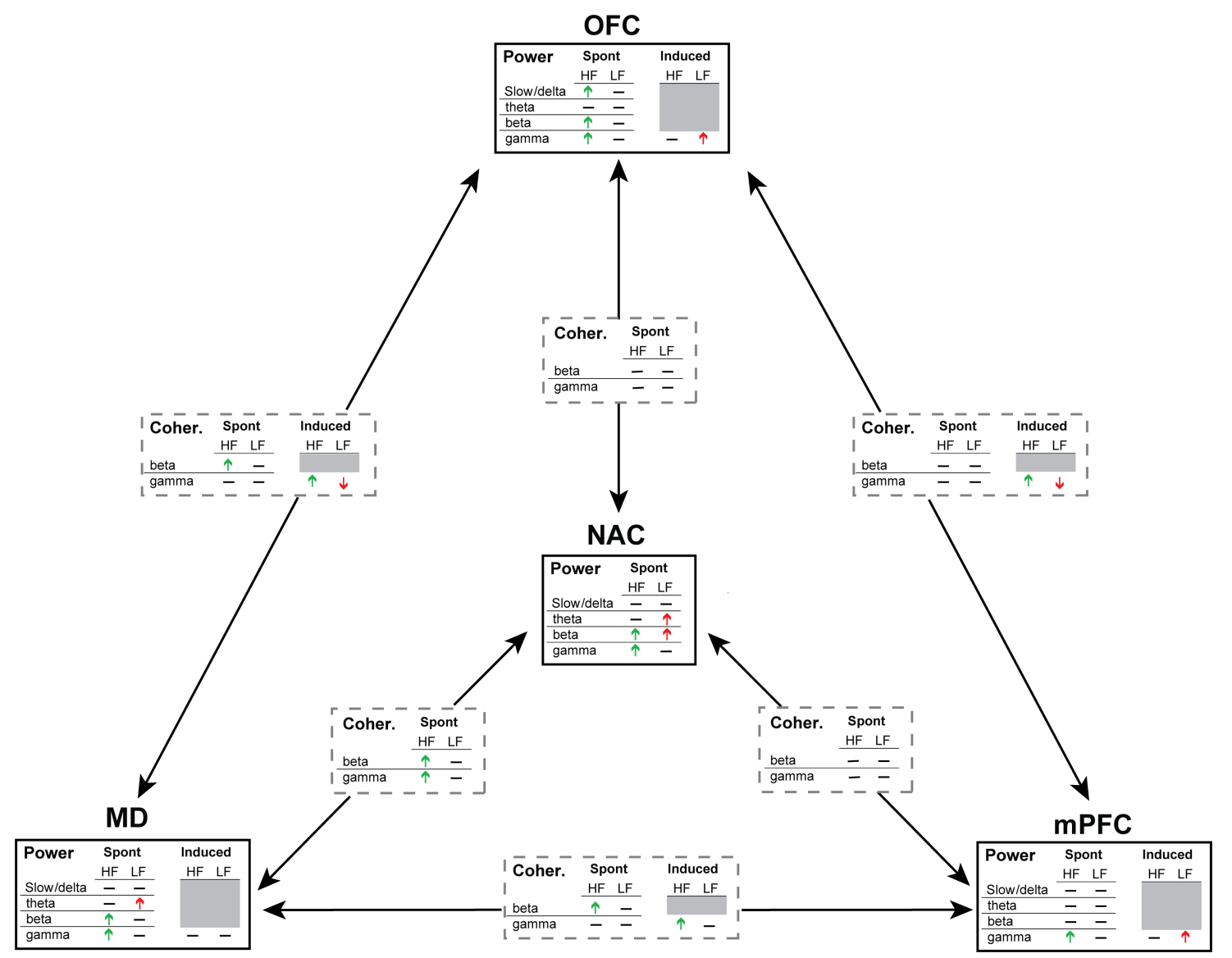

Figure 8. Summary of changes in LFP power and coherence produced by high (HF) and low (LF) frequency NAC DBS. Upward and downward arrows indicate significant increases or decreases, respectively, in power or coherence compared with SHAM DBS. No significant changes were observed in slow/delta or theta coherence between any regions under any stimulation condition; for clarity these data have been omitted the figure. Horizontal line, No change; gray boxes, not tested.

ing of stimuli. Acute NAC stimulation induced gamma oscillations in afferent regions; we therefore assessed the effects of DBS on this induced activity. Induced gamma activity in OFC and $\mathrm{mPFC}$ was decreased compared with prestimulus baseline in the SHAM and HF groups, but remained elevated with LF DBS, possibly representing another correlate of a pathological state. HF DBS enhanced induced gamma coherence between all regions compared with SHAM and LF DBS; conversely, LF DBS decreased induced gamma coherence between OFC-PFC and OFC-MD compared with SHAM. Thus, the effects produced by HF and LF DBS both were different from SHAM but opposite in directions; this situation is analogous to the therapeutic versus deleterious effects produced by HF and LF stimulation, respectively, in the clinic.

\section{DBS effects on evoked LFP response amplitude}

Acute stimulation of the NAC DBS electrode produced shortlatency large-amplitude LFP responses in OFC and mPFC. We showed previously that the initial negative-going component of this evoked potential (N1) is glutamate-dependent and likely stems from synchronous activation of corticostriatal recurrent collaterals; this response was potentiated following $30 \mathrm{~min}$ of $\mathrm{HF}$ NAC DBS in a NMDA-dependent manner (McCracken and Grace, 2007). We report here that this potentiation was unchanged following 60 and 90 min of HF DBS; SHAM or LF DBS did not potentiate N1 amplitude. Evoked responses in mPFC were qualitatively similar, suggesting a mechanism similar to OFC responses; however, $\mathrm{N} 1$ amplitude in $\mathrm{mPFC}$ did not change with any stimulation condition. As with changes in slow oscillations, the lack of potentiation of mPFC evoked responses is likely due to either less activation of $\mathrm{mPFC} \rightarrow \mathrm{NAC}$ axons, intrinsic differences in $\mathrm{mPFC}$ and $\mathrm{OFC}$ anatomy or function, or active suppression of mPFC activity by OFC.

\section{Implications}

The widespread and specific alterations in synchronous LFP activity produced by HF NAC DBS provide further evidence that the therapeutic actions of DBS depend on considerably more than activity in the stimulated nucleus; indeed, our results point to a constellation of changes across an entire corticostriatothalamic circuit. Moreover, these data provide a potential physiological underpinning to the frequency-dependent effects of DBS observed in the clinic. Our previous studies showed that NAC DBS decreased OFC pyramidal cell firing by driving recurrent inhibition; the present work demonstrated widespread increases in synchronous activity known to be mediated through interneurons, such that the therapeutically relevant effects of DBS may not be due to inhibition per se, but to enhanced synchronization of inhibition across cortical and subcortical networks. Increased interneuron-mediated rhythmic gating processes and enhanced synchronous inhibition in afferent structures represent potential mechanisms by which NAC DBS may reduce the pathological metabolic hyperactivity and reintroduce normalized oscillatory activity in prefrontal subregions associated with OCD and depression. 


\section{References}

Barth DS, MacDonald KD (1996) Thalamic modulation of high-frequency oscillating potentials in auditory cortex. Nature 383:78-81.

Benazzouz A, Gao DM, Ni ZG, Piallat B, Bouali-Benazzouz R, Benabid AL (2000) Effect of high-frequency stimulation of the subthalamic nucleus on the neuronal activities of the substantia nigra pars reticulata and ventrolateral nucleus of the thalamus in the rat. Neuroscience 99:289-295.

Beurrier C, Bioulac B, Audin J, Hammond C (2001) High-frequency stimulation produces a transient blockade of voltage-gated currents in subthalamic neurons. J Neurophysiol 85:1351-1356.

Boraud T, Bezard E, Bioulac B, Gross C (1996) High frequency stimulation of the internal globus pallidus (GPi) simultaneously improves parkinsonian symptoms and reduces the firing frequency of GPi neurons in the MPTP-treated monkey. Neurosci Lett 215:17-20.

Bressler SL (1984) Spatial organization of EEGs from olfactory bulb and cortex. Electroencephalogr Clin Neurophysiol 57:270-276.

Buzsáki G (2002) Theta oscillations in the hippocampus. Neuron 33:325-340.

Cooper SE, Kuncel AM, Wolgamuth BR, Rezai AR, Grill WM (2008) A model predicting optimal parameters for deep brain stimulation in essential tremor. J Clin Neurophysiol 25:265-273.

Cowan RL, Wilson CJ (1994) Spontaneous firing patterns and axonal projections of single corticostriatal neurons in the rat medial agranular cortex. J Neurophysiol 71:17-32.

Crombag HS, Badiani A, Maren S, Robinson TE (2000) The role of contextual versus discrete drug-associated cues in promoting the induction of psychomotor sensitization to intravenous amphetamine. Behav Brain Res 116:1-22.

Deckersbach T, Dougherty DD, Rauch SL (2006) Functional imaging of mood and anxiety disorders. J Neuroimaging 16:1-10.

DeCoteau WE, Thorn C, Gibson DJ, Courtemanche R, Mitra P, Kubota Y, Graybiel AM (2007) Oscillations of local field potentials in the rat dorsal striatum during spontaneous and instructed behaviors. J Neurophysiol 97:3800-3805.

Desarkar P, Sinha VK, Jagadheesan K, Nizamie SH (2007) Subcortical functioning in obsessive-compulsive disorder: an exploratory EEG coherence study. World J Biol Psychiatry 8:196-200.

Evans KC, Dougherty DD, Pollack MH, Rauch SL (2006) Using neuroimaging to predict treatment response in mood and anxiety disorders. Ann Clin Psychiatry 18:33-42.

Farmer SF (1998) Rhythmicity, synchronization and binding in human and primate motor systems. J Physiol 509:3-14.

Faulkner HJ, Traub RD, Whittington MA (1999) Anaesthetic/amnesic agents disrupt beta frequency oscillations associated with potentiation of excitatory synaptic potentials in the rat hippocampal slice. Br J Pharmacol 128:1813-1825.

Flaherty AW, Williams ZM, Amirnovin R, Kasper E, Rauch SL, Cosgrove GR, Eskandar EN (2005) Deep brain stimulation of the anterior internal capsule for the treatment of Tourette syndrome: technical case report. Neurosurgery 57:E403; discussion E403.

Florin E, Reck C, Burghaus L, Lehrke R, Gross J, Sturm V, Fink GR, Timmermann L (2008) Ten Hertz thalamus stimulation increases tremor activity in the subthalamic nucleus in a patient with Parkinson's disease. Clin Neurophysiol 119:2098-2103.

Gabbott PL, Warner TA, Jays PR, Salway P, Busby SJ (2005) Prefrontal cortex in the rat: projections to subcortical autonomic, motor, and limbic centers. J Comp Neurol 492:145-177.

Greenberg BD, Gabriels LA, Malone DA Jr, Rezai AR, Friehs GM, Okun MS, Shapira NA, Foote KD, Cosyns PR, Kubu CS, Malloy PF, Salloway SP, Giftakis JE, Rise MT, Machado AG, Baker KB, Stypulkowski PH, Goodman WK, Rasmussen SA, Nuttin BJ (2009) Deep brain stimulation of the ventral internal capsule/ventral striatum for obsessive-compulsive disorder: worldwide experience. Mol Psychiatry. Advance online publication. Retrieved April 6, 2009. doi:10.1038/mp.2008.55.

Haenschel C, Baldeweg T, Croft RJ, Whittington M, Gruzelier J (2000) Gamma and beta frequency oscillations in response to novel auditory stimuli: a comparison of human electroencephalogram (EEG) data with in vitro models. Proc Natl Acad Sci U S A 97:7645-7650.

Hajós M, Hoffmann WE, Kocsis B (2008) Activation of cannabinoid-1 receptors disrupts sensory gating and neuronal oscillation: relevance to schizophrenia. Biol Psychiatry 63:1075-1083.

Hammond C, Ammari R, Bioulac B, Garcia L (2008) Latest view on the mechanism of action of deep brain stimulation. Mov Disord 23:2111-2121.

Homayoun H, Moghaddam B (2006) Progression of cellular adaptations in medial prefrontal and orbitofrontal cortex in response to repeated amphetamine. J Neurosci 26:8025-8039.

Kamondi A, Acsády L, Wang XJ, Buzsáki G (1998) Theta oscillations in somata and dendrites of hippocampal pyramidal cells in vivo: activitydependent phase-precession of action potentials. Hippocampus 8:244-261.

Karadag F, Oguzhanoglu NK, Kurt T, Oguzhanoglu A, Ateşci F, Ozdel O (2003) Quantitative EEG analysis in obsessive compulsive disorder. Int J Neurosci 113:833-847.

Kisley MA, Cornwell ZM (2006) Gamma and beta neural activity evoked during a sensory gating paradigm: effects of auditory, somatosensory and cross-modal stimulation. Clin Neurophysiol 117:2549-2563.

Kiss ZH, Mooney DM, Renaud L, Hu B (2002) Neuronal response to local electrical stimulation in rat thalamus: physiological implications for mechanisms of deep brain stimulation. Neuroscience 113:137-143.

Kopell N, Ermentrout GB, Whittington MA, Traub RD (2000) Gamma rhythms and beta rhythms have different synchronization properties. Proc Natl Acad Sci U S A 97:1867-1872.

Kuhn J, Lenartz D, Huff W, Lee S, Koulousakis A, Klosterkoetter J, Sturm V (2007a) Remission of alcohol dependency following deep brain stimulation of the nucleus accumbens: valuable therapeutic implications? J Neurol Neurosurg Psychiatry 78:1152-1153.

Kuhn J, Lenartz D, Mai JK, Huff W, Lee SH, Koulousakis A, Klosterkoetter J, Sturm V (2007b) Deep brain stimulation of the nucleus accumbens and the internal capsule in therapeutically refractory Tourette-syndrome. J Neurol 254:963-965.

Kuncel AM, Cooper SE, Wolgamuth BR, Grill WM (2007) Amplitude- and frequency-dependent changes in neuronal regularity parallel changes in tremor With thalamic deep brain stimulation. IEEE Trans Neural Syst Rehabil Eng 15:190-197.

Kupsch A, Klaffke S, Kühn AA, Meissner W, Arnold G, Schneider GH, MaierHauff K, Trottenberg T (2003) The effects of frequency in pallidal deep brain stimulation for primary dystonia. J Neurol 250:1201-1205.

Kuskowski MA, Malone SM, Kim SW, Dysken MW, Okaya AJ, Christensen KJ (1993) Quantitative EEG in obsessive-compulsive disorder. Biol Psychiatry 33:423-430.

Leocani L, Locatelli M, Bellodi L, Fornara C, Hénin M, Magnani G, Mennea S, Comi G (2001) Abnormal pattern of cortical activation associated with voluntary movement in obsessive-compulsive disorder: an EEG study. Am J Psychiatry 158:140-142.

Locatelli M, Bellodi L, Grassi B, Scarone S (1996) EEG power modifications in obsessive-compulsive disorder during olfactory stimulation. Biol Psychiatry 39:326-331.

Logothetis NK (2003) The underpinnings of the BOLD functional magnetic resonance imaging signal. J Neurosci 23:3963-3971.

Logothetis NK, Pauls J, Augath M, Trinath T, Oeltermann A (2001) Neurophysiological investigation of the basis of the fMRI signal. Nature 412:150-157.

Maier A, Wilke M, Aura C, Zhu C, Ye FQ, Leopold DA (2008) Divergence of fMRI and neural signals in V1 during perceptual suppression in the awake monkey. Nat Neurosci 11:1193-1200.

Malone DA Jr, Dougherty DD, Rezai AR, Carpenter LL, Friehs GM, Eskandar EN, Rauch SL, Rasmussen SA, Machado AG, Kubu CS, Tyrka AR, Price LH, Stypulkowski PH, Giftakis JE, Rise MT, Malloy PF, Salloway SP, Greenberg BD (2009) Deep brain stimulation of the ventral capsule/ ventral striatum for treatment-resistant depression. Biol Psychiatry 65:267-275.

McCracken CB, Grace AA (2007) High-frequency deep brain stimulation of the nucleus accumbens region suppresses neuronal activity and selectively modulates afferent drive in rat orbitofrontal cortex in vivo. J Neurosci 27:12601-12610.

McIntyre CC, Savasta M, Kerkerian-Le Goff L, Vitek JL (2004) Uncovering the mechanism(s) of action of deep brain stimulation: activation, inhibition, or both. Clin Neurophysiol 115:1239-1248.

Mitra PP, Pesaran B (1999) Analysis of dynamic brain imaging data. Biophys J 76:691-708.

Mitzdorf U (1985) Current source-density method and application in cat cerebral cortex: investigation of evoked potentials and EEG phenomena. Physiol Rev 65:37-100. 
Moghaddam B, Homayoun H (2008) Divergent plasticity of prefrontal cortex networks. Neuropsychopharmacology 33:42-55.

Moro E, Esselink RJ, Xie J, Hommel M, Benabid AL, Pollak P (2002) The impact on Parkinson's disease of electrical parameter settings in STN stimulation. Neurology 59:706-713.

Nandi D, Jenkinson N, Stein J, Aziz T (2008) The pedunculopontine nucleus in Parkinson's disease: primate studies. Br J Neurosurg 22 [Suppl 1]:S4-S8.

Neville KR, Haberly LB (2003) Beta and gamma oscillations in the olfactory system of the urethane-anesthetized rat. J Neurophysiol 90:3921-3930.

Nowak LG, Bullier J (1998a) Axons, but not cell bodies, are activated by electrical stimulation in cortical gray matter. I. Evidence from chronaxie measurements. Exp Brain Res 118:477-488.

Nowak LG, Bullier J (1998b) Axons, but not cell bodies, are activated by electrical stimulation in cortical gray matter. II. Evidence from selective inactivation of cell bodies and axon initial segments. Exp Brain Res 118:489-500

Paz R, Bauer EP, Paré D (2008) Theta synchronizes the activity of medial prefrontal neurons during learning. Learn Mem 15:524-531.

Pesaran B, Pezaris JS, Sahani M, Mitra PP, Andersen RA (2002) Temporal structure in neuronal activity during working memory in macaque parietal cortex. Nat Neurosci 5:805-811.

Pogarell O, Juckel G, Mavrogiorgou P, Mulert C, Folkerts M, Hauke W, Zaudig M, Möller HJ, Hegerl U (2006) Symptom-specific EEG power correlations in patients with obsessive-compulsive disorder. Int J Psychophysiol 62:87-92.

Ranck JB Jr (1975) Which elements are excited in electrical stimulation of mammalian central nervous system: a review. Brain Res 98:417-440.

Rauch SL, Dougherty DD, Malone D, Rezai A, Friehs G, Fischman AJ, Alpert NM, Haber SN, Stypulkowski PH, Rise MT, Rasmussen SA, Greenberg BD (2006) A functional neuroimaging investigation of deep brain stimulation in patients with obsessive-compulsive disorder. J Neurosurg 104:558-565.

Roopun AK, Middleton SJ, Cunningham MO, LeBeau FE, Bibbig A, Whittington MA, Traub RD (2006) A beta2-frequency $(20-30 \mathrm{~Hz})$ oscillation in nonsynaptic networks of somatosensory cortex. Proc Natl Acad Sci U S A 103:15646-15650.

Sanchez-Vives MV, McCormick DA (2000) Cellular and network mechanisms of rhythmic recurrent activity in neocortex. Nat Neurosci 3:1027-1034.

Schlaepfer TE, Cohen MX, Frick C, Kosel M, Brodesser D, Axmacher N, Joe AY, Kreft M, Lenartz D, Sturm V (2008) Deep brain stimulation to reward circuitry alleviates anhedonia in refractory major depression. Neuropsychopharmacology 33:368-377.
Schnitzler A, Gross J (2005) Normal and pathological oscillatory communication in the brain. Nat Rev Neurosci 6:285-296.

Serra FP, Palma V, Nolfe G, Buscaino GA (1994) An electrophysiological study in obsessional compulsive disorders. Acta Neurol (Napoli) $16: 240-248$.

Shu Y, Hasenstaub A, McCormick DA (2003) Turning on and off recurrent balanced cortical activity. Nature 423:288-293.

Singer W (1993) Synchronization of cortical activity and its putative role in information processing and learning. Annu Rev Physiol 55:349-374.

Steriade M (2006) Grouping of brain rhythms in corticothalamic systems. Neuroscience 137:1087-1106.

Steriade M, Nuñez A, Amzica F (1993) A novel slow ( $<1 \mathrm{~Hz}$ ) oscillation of neocortical neurons in vivo: depolarizing and hyperpolarizing components. J Neurosci 13:3252-3265.

Sturm V, Lenartz D, Koulousakis A, Treuer H, Herholz K, Klein JC, Klosterkötter J (2003) The nucleus accumbens: a target for deep brain stimulation in obsessive-compulsive- and anxiety-disorders. J Chem Neuroanat 26:293-299.

Tallon-Baudry C (2003) Oscillatory synchrony and human visual cognition. J Physiol Paris 97:355-363.

Timmermann L, Wojtecki L, Gross J, Lehrke R, Voges J, Maarouf M, Treuer H, Sturm V, Schnitzler A (2004) Ten-Hertz stimulation of subthalamic nucleus deteriorates motor symptoms in Parkinson's disease. Mov Disord 19:1328-1333.

Traub RD, Whittington MA, Buhl EH, Jefferys JG, Faulkner HJ (1999) On the mechanism of the gamma $\rightarrow$ beta frequency shift in neuronal oscillations induced in rat hippocampal slices by tetanic stimulation. J Neurosci 19:1088-1105.

Traub RD, Bibbig A, LeBeau FE, Buhl EH, Whittington MA (2004) Cellular mechanisms of neuronal population oscillations in the hippocampus in vitro. Annu Rev Neurosci 27:247-278.

van Kuyck K, Demeulemeester H, Feys H, De Weerdt W, Dewil M, Tousseyn T, De Sutter P, Gybels J, Bogaerts K, Dom R, Nuttin B (2003) Effects of electrical stimulation or lesion in nucleus accumbens on the behaviour of rats in a T-maze after administration of 8-OH-DPAT or vehicle. Behav Brain Res 140:165-173.

Vitek JL (2002) Mechanisms of deep brain stimulation: excitation or inhibition. Mov Disord 17 [Suppl 3]:S69-S72.

Womelsdorf T, Fries P, Mitra PP, Desimone R (2006) Gamma-band synchronization in visual cortex predicts speed of change detection. Nature 439:733-736.

Yamawaki N, Stanford IM, Hall SD, Woodhall GL (2008) Pharmacologically induced and stimulus evoked rhythmic neuronal oscillatory activity in the primary motor cortex in vitro. Neuroscience 151:386-395. 